\title{
池塘养殖环境下滇池金线鲃仔稚鱼的食性转化与生长
}

\author{
潘晓赋，杨君兴*，李再云，陈小勇 \\ (中国科学院昆明动物研究所, 云南昆明 650223)
}

\begin{abstract}
摘要: 2008 年 3 月一 2009 年 3 月, 把 35000 尾滇池金线鲃(Sinocyclocheilus grahami)仔稚鱼分别饲养于 3 个 池塘进行对比饲养研究。密度分别是: $\mathrm{P}_{1}$ 为 208 尾 $/ \mathrm{m}^{3} ; \mathrm{P}_{2}$ 为 167 尾 $/ \mathrm{m}^{3} ; \mathrm{P}_{3}$ 为 41 尾 $/ \mathrm{m}^{3}$ 。饲养期间采用相同的管理 措施。每天数据收集采用: 在仔鱼睬化后的 5 16d 的每天 8:00 和 18:00 从每个育苗池中捞取 5 8 尾仔鱼进行测 量分析。仔鱼开口 $30 \mathrm{~d}$ 后, 每月从培育池各捞取 15 尾, 测量全长和体重。研究发现, 滇池金线鲃仔鱼的开口时 间为瞬化后的 $5 \mathrm{~d}$, 混合营养期 $3 \sim 5 \mathrm{~d}$, 这与初睬的滇池金线鲃仔鱼的全长和卵黄囊较大有关。在容易造成仔鱼 大量死亡的混合营养期, 通过采用浆状物一轮虫一人工混合饲料的方式, 大幅度提高了滇池金线鲃仔稚鱼的存活 率，䁔化一年后的滇池金线鲃稚鱼存活率可达 $82.7 \%$ 。经过一周年的饲养，滇池金线鲃稚鱼的体重由 $(0.027 \pm 0.01)$ $\mathrm{g}(0.003 \sim 1.22)$ 增长到 $(8.83 \pm 0.54) \mathrm{g}(3.7 \sim 16.7)$, 月均增重为 $0.73 \mathrm{~g}$; 全长由 $(15.8 \pm 0.30) \mathrm{mm}(11.5 \sim 20.0)$ 增长到 $(91.6 \pm 1.67) \mathrm{mm}(75.0 \sim 16.0)$, 全长月均增长 $6.32 \mathrm{~mm}$ 。滇池金线鲃稚鱼全年全长的增长呈线性相关, $y=9.82+7.05$ month $\left(R^{2}=0.9891\right)$; 全年体重的增长呈指数关系, $y=0.0158$ month $^{2.54}\left(R^{2}=0.9939\right)$ 。
\end{abstract}

关键词: 滇池金线鲃; 食性转换; 生长; 仔稚鱼

中图分类号: Q959.468; Q959.468.05 文献标识码: A 文章编号: 0254-5853-(2009)04-0433-05

\section{Feeding Changes and Growth Performance of Sinocyclocheilus grahami (Pisces, Barbinae) Larvae and Juveniles in Farm Environment}

\author{
PAN Xiao-fu, YANG Jun-xing*, LI Zai-yun, CHEN Xiao-yong \\ (Kunming Institute of Zoology, the Chinese Academy of Sciences, Kunming 650223, China)
}

\begin{abstract}
From March 2008 to March 2009, comparative observations of feeding and growth were undertaken on 35,000 larvae cultivated in 3 pools, with respective densities of $P_{1} 208$ larvae $/ \mathrm{m}^{3} ; \mathrm{P}_{2} 167$ larvae $/ \mathrm{m}^{3} ; \mathrm{P}_{3} 41$ larvae $/ \mathrm{m}^{3}$. Identical daily management processes were applied to the 3 pools. Data was collected on 5-8 larvae everyday at 08:00 and 18:00 within 15 days of hatching. 30 days from first dieting, the total length and body weight were measured on 15 juveniles collected from each pool. The first dieting of $S$. grahami larvae occurred in the $5^{\text {th }}$ day after hatching, with a mixed nutrition phase of 3-5 days. The larvae within mixed nutrition conditions were vulnerable to death. In order to promote the survival in mixed nutrition, the larvae and juveniles were raised with jelly-rotifers-compound feed. The mean survival rate of juveniles S. grahami was $82.7 \%$. After one year of cultivation, body weight increased from $(0.027 \pm 0.01)$ $\mathrm{g}(0.003-1.22)$ to $(8.83 \pm 0.54) \mathrm{g}(3.7-16.7)$, net growth being $0.73 \mathrm{~g}$ per month. Total length increased from $15.8 \pm 0.30$ $\mathrm{mm}(11.5-20.0)$ to $91.6 \pm 1.67 \mathrm{~mm}(75.0-116.0)$, net growth being $6.32 \mathrm{~mm}$ per month. Linear growth in total length can be described by the equation: $y=9.82+7.05$ month $\left(R^{2}=0.9891\right)$. Index growth in body weight can be described by equation: $y=0.0158$ month $^{2.54}\left(R^{2}=0.9939\right)$.
\end{abstract}

Key words: Sinocyclocheilus grahami; Feeding changes; Growth performance; Larvae and Juveniles 在仔稚的生长过程中, 天然食物是仔稚鱼存活

和生长的营养基础。大多数鱼类在仔稚鱼阶段的适

收稿日期：2009-06-16; 接受日期：2009-07-16

基金项目：国家重点基础研究发展计划(2007CB411600); 全球环境基金会/世界银行项目(GEF-MSP grant No. TF051795); 云南省发展和改 革委员会项目; 中国科学院西部之光项目(292006312D11033); 云南省科技厅社会发展科技计划(2008CA001)

*通讯作者 (Corresponding author), E-mail: yangjx@mail.kiz.ac.cn

第一作者简介: 潘晓赋 (1976-), 男, 硕士, 助理研究员, 主要从事水产学和鱼类保护研究 
口食物资源通常比较少, 而且大都需要活饵。不同 发育阶段的仔鱼, 其生活方式、营养状况和生长类 型不同, 致使其对食物进行选择。在饲养环境下, 由于高密度饲养导致天然食物远远不能满足仔稚 鱼营养需要, 其后果是仔稚鱼缺乏适口的食饵而大 量死亡 (Yin, 1995)。鲇(Silurus asotus)和线纹尖塘 鳢(Oxyeleotris lineolatus)仔鱼在开口阶段, 如果缺 乏适合的食饵, 会造成仔鱼大量死亡 (Qiao et al, 2007; Zhang et al, 2007)。通常认为, 食性转化是 仔稚鱼对环境、行为和摄食模式等变化的一种前期 准备 (Youson, 1988)。

历史上, 滇池金线鲃(Sinocyclocheilus grahami) 是滇池沿岸渔民的主要捕获对象。后来受外来物种 的引入、水质污染、过度捕捞等因素的影响, 导致 滇池金线鲃数量急剧减少 (Chen et al, 2001)。该 物种于 1989 年被列为国家二级保护动物, 在《中

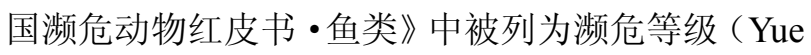
\& Chen, 1998)。根据我们最新调查, 滇池金线鲃 种群衰退十分严重, 其种群在滇池湖体已经灭绝, 目前仅在滇池沿岸的 20 个龙潭和牧羊河有少量居 群分布 (Pan et al, 2009)。作为云南珍贵的食用鱼 类, 大量人工繁殖和饲养是防止其灭绝, 增加其种 群数量的主要办法。因此, 滇池金线鲃人工驯化已 经被关注多年, 在人工驯化的过程中, 难以突破的 技术瓶颈是人工繁殖。中国科学院昆明动物研究所 珍稀鱼类保育研究基地于 2007 年首次成功实现滇 池金线鲃人工繁殖 (Yang et al, 2007)。本项研究 通过对滇池金线鲃仔稚鱼的食性转化与生长特点 的分析, 为今后制定和完善仔稚鱼的培育方案提供 基础素材, 提高仔稚鱼的品质和存活率。

\section{1 材料与方法}

\section{1 鱼苗的来源与管理}

滇池金线鲃鱼苗系 2008 年 3 月中国科学院昆 明动物研究所珍稀鱼类保育研究基地通过人工繁 殖获得。亲本系 2005 年 5-7 月采集自昆明嵩明牧 羊河。催产素雌鱼剂量按每千克体重 $1 \mathrm{mg}$ 马来酸地 欧酮 $+1 \mu \mathrm{g}$ 促黄体素释放激素 $\mathrm{A}_{2}$, 用 $0.9 \%$ 的生理盐 水稀释混合后, 肌肉注射。注射后 $24 \mathrm{~h}$ 左右施行人 工助产、干法受精 (Yang et al, 2007)。仔鱼孵化 出 $4 \mathrm{~d}$ 后, 开始移入育苗池, 滇池金线鲃鱼苗的日常 管理参照鲤鱼鱼苗管理方法 (Horváth et al, 2002)。
使用 3 个育苗池分别为 $\mathrm{P}_{1}\left(20 \mathrm{~m}^{2}\right.$; 水泥底, 水 深 $1.2 \mathrm{~m}) 、 \mathrm{P}_{2}\left(50 \mathrm{~m}^{2}\right.$; 水泥底, 水深 $\left.1.2 \mathrm{~m}\right)$ 和 $\mathrm{P}_{3}(330$ $\mathrm{m}^{2}$; 泥底, 水深 $1.5 \mathrm{~m}$ )。密度分别是: $\mathrm{P}_{1}$ 为 208 尾 $/ \mathrm{m}^{3} 、 \mathrm{P}_{2}$ 为 167 尾 $/ \mathrm{m}^{3} 、 \mathrm{P}_{3}$ 为 41 尾 $/ \mathrm{m}^{3}$ 。使用前用高 锰酸钾全池泼洒消毒, 然后注入新鲜水。所有池子 用黑色遮光网遮盖全池约二分之一, 避免阳光直 射。池内每 $10 \mathrm{~m}^{2}$ 布置一个充气沙头充气, 增加池 内溶氧, 保持实时溶氧 $>5 \mathrm{mg} / \mathrm{L}$ 。水温保持在 $16 \sim 18^{\circ} \mathrm{C}$ 。每隔 3 $5 \mathrm{~d}$ 注入新水一次, 每次增加池水 $15 \sim 20 \mathrm{~cm}$ 。

\section{2 数据收集}

2008 年 3 月一 2009 年 3 月, 在仔鱼孵化后 5 16 $\mathrm{d}$ 的每天 8:00 和 18:00 从每个育苗池中捞取 5 8 尾仔鱼进行消化道充塞度分析。具体方法为首先使 用 SF2000 数显游标卡尺 (桂林广陆数字测控股份 有限公司）测量仔鱼的全长（TL，精度 $0.01 \mathrm{~mm}$ ), 随后用百灵 LA204 电子天平 (常熟市衡器厂) 测量 仔鱼的体重（BW，精度 $1 \mathrm{mg}$ )。仔鱼开口 $30 \mathrm{~d}$ 后, 每月从培育池各捞取 15 尾, 测量全长和体重。在 饲养期间，记录管理日志。

\section{3 数据处理}

充塞度分级方法: 0 级, 消化道内无食物; 1 级, 消化道含 $0 \%$ 25\%的食物; 2 级, 消化道含 $25 \% \sim 50 \%$ 的食物; 3 级, 消化道含 $50 \% \sim 75 \%$ 的食物; 4 级, 消化道含 $75 \%$ 100\%的食物; 5 级, 整个消 化道充满食物。特定生长率(specific growth rate, $\mathrm{SGR}): S G R=\left[\left(\ln W_{2}-\ln W_{1}\right) /\left(\mathrm{t}_{2}-\mathrm{t}_{1}\right)\right] \times 100$; 式中 $W_{1}$ 和 $W_{2}$ 是初始体重和最终体重 $(\mathrm{g}), t$ 是试验时间 (Zhang et al，2008)。

各测量数据均采用平均数土标准误 $($ mean $\pm S E)$ 。 用单因素方差分析和回归分析比较分析处理数据, 所有数据处理和制图均在 Sigmaplot 2001 中进行。 显著性水平为 $P<0.05$ 。

\section{2 结 果}

\section{1 仔鱼的食性转化与饲养}

仔鱼最早开口时间是孵化后的第 $5 \mathrm{~d}$, 比仔鱼 卵黄耗尽早 $1 \sim 2 \mathrm{~d}$, 消化道充塞度为 0 级的个体占 $86.4 \%$ 。至孵化后第 $7 \mathrm{~d}$, 充塞度为 0 级者下降至 $8.3 \%$, 以后基本维持在 $3 \%$ 左右。滇池金线鲃仔鱼 由内源营养转换为外源营养前, 有 3 5d 的混合营 养期。池塘养殖的仔鱼, 出膜后几天内在育苗池的 池壁四周水面，尤其是鱼池的四个角落集聚分布; 
随着卵黄囊的消耗, 仔鱼开始有摄食活动, 活动范 围扩大到鱼池的四壁和池底; 受食能力进一步提高 后, 仔鱼主动集聚在浮游动物密集的区域, 摄食大 小合适的活饵。在仔鱼进入稚鱼发育前, 仔鱼开始 摄食人工混合饵料, 而且摄食强度逐渐增强。

鱼苗开口后的 5 10 d, 每日投喂 4 次, 投喂的 饵料分别为: 蛋黄浆、豆浆等, 具体投喂量为每千 尾仔鱼按蛋黄半个、干黄豆 $30 \mathrm{~g}$ 研磨成浆全池泼酒。 鱼苗开口后的 8 20d, 每日投喂轮虫 2 次, 轮虫投 喂量为 200 万 300 万个 $/ \mathrm{m}^{3}$ 。鱼苗开口后的 $40 \mathrm{~d}$ 以 后, 开始投喂破碎的 111 鲤鱼种鱼饲料（颗粒直径 $\leq 0.5 \mathrm{~mm}$ ，通威股份有限公司昆明分公司), 每日投 喂量按 $2 \% \sim 5 \%$ 饵料系数的计算投喂量, 并根据水 温和鱼的进食情况酌情加减。孵化后第 $10 \mathrm{~d}$ 开始从 仔鱼的消化道内检查出轮虫, 第 $18 \mathrm{~d}$ 检查到枝角类, 第 $43 \mathrm{~d}$ 检查到人工配合饲料的食糜。捊化后 $35 \sim 45 \mathrm{~d}$, 鳞片开始出现, 仔鱼进入稚鱼发育。

\section{2 仔鱼的生长}

孵化后 5 16 d 滇池金线鲃仔鱼的体重由 $(1.93 \pm 0.59) \mathrm{mg}) （ 1.0 \sim 3)$ 增长到 $(24.7 \pm 0.74) \mathrm{mg}$ (19.0 29.0), 日均增重为 $1.90 \mathrm{mg}$; 全长由

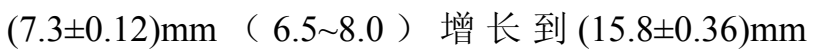
(12.5 17.5), 全长日均增长 $0.71 \mathrm{~mm}$ 。孵化后 16 天CF为 0.9636 , SGR为 $23.17 \%$ 。但在此期间, 3 个 培育池仔鱼每日全长 $\left(F_{(2,33)}=0.009, P>0.05\right)$ 和 体重 $\left(F_{(2,33)}=0.0039, P>0.05\right)$ 不存在显著差异。 全长与餒化后天数呈线性相关 $\left(R^{2}=0.9830\right.$, 图 1); 体重与捊化后天数呈指数函数关系 $\left(R^{2}=0.9760\right.$, 图 1)。

\section{3 稚鱼的生长}

滇池金线鲃稚鱼的体重由 $(0.027 \pm 0.01) \mathrm{g}$ (0.003 1.22) 增长到(8.83 \pm 0.54$) \mathrm{g}$ (3.7 16.7), 月 均增重为 $0.73 \mathrm{~g}$; 全长由 $(15.8 \pm 0.30) \mathrm{mm}(11.5 \sim 20.0)$ 增长到(91.6 \pm 1.67$) \mathrm{mm}$ (75.0 116.0), 全长月均增长 $6.32 \mathrm{~mm}$ 。滇池金线鲃稚鱼全年全长的增长呈线性相 关, $y=9.82+7.05$ month $\left(R^{2}=0.9891\right)$; 全年体重的 增长呈指数关系, $y=0.0158 \mathrm{month}^{2.54}\left(R^{2}=0.9939\right)$ 。

\section{4 不同养殖环境对稚鱼生长的影响}

在试验结束时, 三个培育池滇池金线鲃稚鱼的 全长、体重、月增长量均以 $\mathrm{P}_{3}$ 最高, $\mathrm{P}_{1}$ 次之, $\mathrm{P}_{2}$ 最 小。体重呈指数增长, 全长呈线形增长 (表 1, 图 2)。 $\mathrm{P}_{1}$ 和 $\mathrm{P}_{2}$ 池之间每月体重和全长的差异均不显著 $(P>0.05) ; P_{1}$ 和 $P_{3}$ 池之间除 10 月份全长、3 月份 全长和体重差异不显著外, 其余月份全长和体重均 存在显著差异 $(P<0.05) ; \mathrm{P}_{2}$ 和 $\mathrm{P}_{3}$ 池之间每月体重和 全长均存在显著差异 $(P<0.05)$ 。

\section{3 讨 论}

\section{1 仔鱼的开口时间与受食能力}

仔鱼开口时间因种而异。鞍带石斑鱼 (Epinephelus lanceol)和线纹尖塘鳢仔鱼的开口时间 为孵化后 $3 \mathrm{~d}$, 鞍带石斑鱼的混合营养期为 $10 \mathrm{~d}$, 而半滑舌鳎(Cynoglossus semilaevis)的混合营养期 仅需 $2 \mathrm{~d}$ 就可以建立完善的外源营养 (Zhuang et al, 2005; Li et al, 2006; Zhang et al，2007)。滇池金 线鲃仔鱼的开口时间为捊化后的第 $5 \mathrm{~d}$, 在开口后 需要及时投喂开口饵料。适当的开口饵料可以保证 仔鱼的较高存活率。如果不及时投喂饵料或提供的 饵料不适口，仔鱼摄取不到充足的营养，会造成仔 鱼的大量死亡或影响后期生长发育。另外，仔鱼的

表 1 从试验开始至结束 3 个培育池滇池金线鲃稚鱼的全长和体重

Tab. 1 Total length and body weight of Sinocyclocheilus grahami juveniles in three ponds at commencement and conclusion of experimentation

\begin{tabular}{llll}
\hline \multicolumn{1}{c}{ 项目 item } & \multicolumn{1}{c}{$\mathrm{P}_{1}$} & \multicolumn{1}{c}{$\mathrm{P}_{2}$} & \multicolumn{1}{c}{$\mathrm{P}_{3}$} \\
\hline 体重 $(\mathrm{g})$ Weight & & & \\
$\quad$ 开始 Begin & $(0.024 \pm 0.003)$ & $(0.027 \pm 0.01)$ & $(0.029 \pm 0.003)$ \\
$\quad$ 结束 End & $(8.53 \pm 0.96)$ & $(7.25 \pm 0.71)$ & $(10.73 \pm 0.91)$ \\
月增长 Net growth of per month & 0.71 & 0.60 & 0.89 \\
增长方程 Growth of equation & $y=0.0069 \mathrm{month}^{2.84}$ & $y=0.0092 \mathrm{month}^{2.68}$ & $y=0.0381 \mathrm{month}^{2.27}$ \\
全长(mm)Total length & & & \\
$\quad$ 开始 Begin & $(15.3 \pm 0.60)$ & $(16.1 \pm 0.46)$ & $(16.1 \pm 0.49-$ \\
$\quad$ 结束 End & $(90.2 \pm 3.017)$ & $(88.2 \pm 2.34)$ & $(96.4 \pm 3.02)$ \\
月增长 Net growth of per month & 6.24 & 6.01 & 6.69 \\
增长方程 Growth of equation & $y=8.06+6.97 \mathrm{month}$ & $y=7.64+6.83$ month & $y=13.75+7.36 \mathrm{month}$ \\
\hline
\end{tabular}




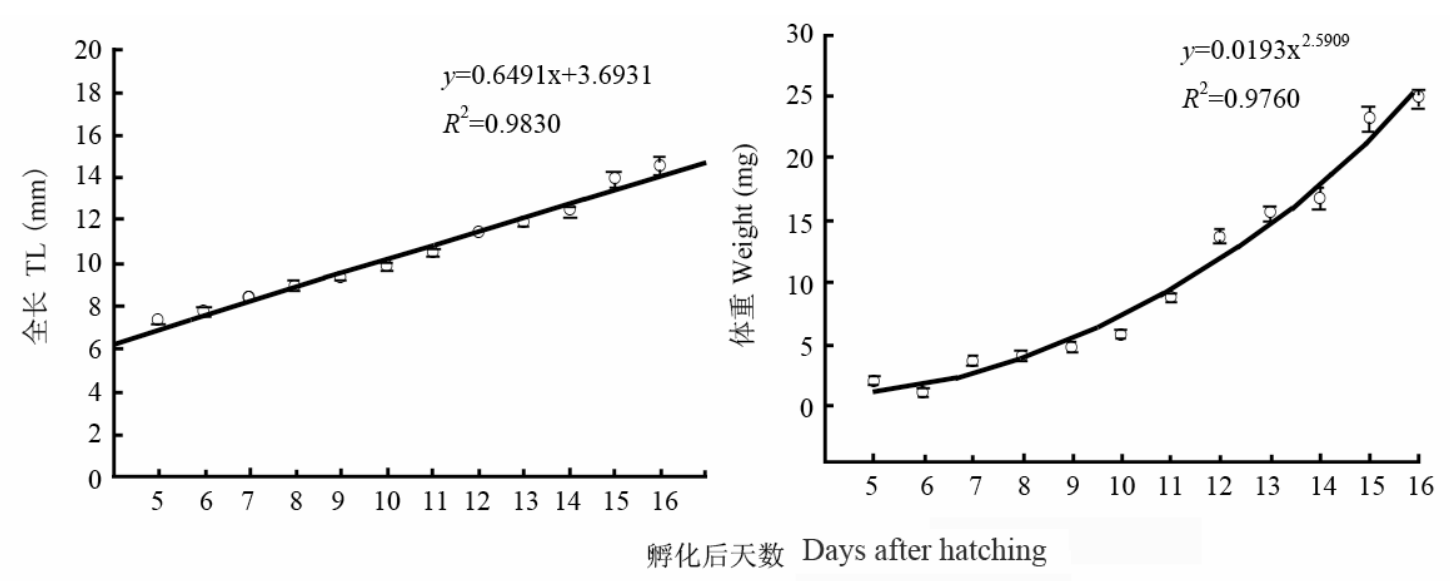

图 1 睬化后 5 16 d 滇池金线鲃仔鱼全长和体重的回归分析

Fig. 1 Regression analyses of total length and weight of Sinocyclocheilus grahami larvae from 5 to 16 days after hatching

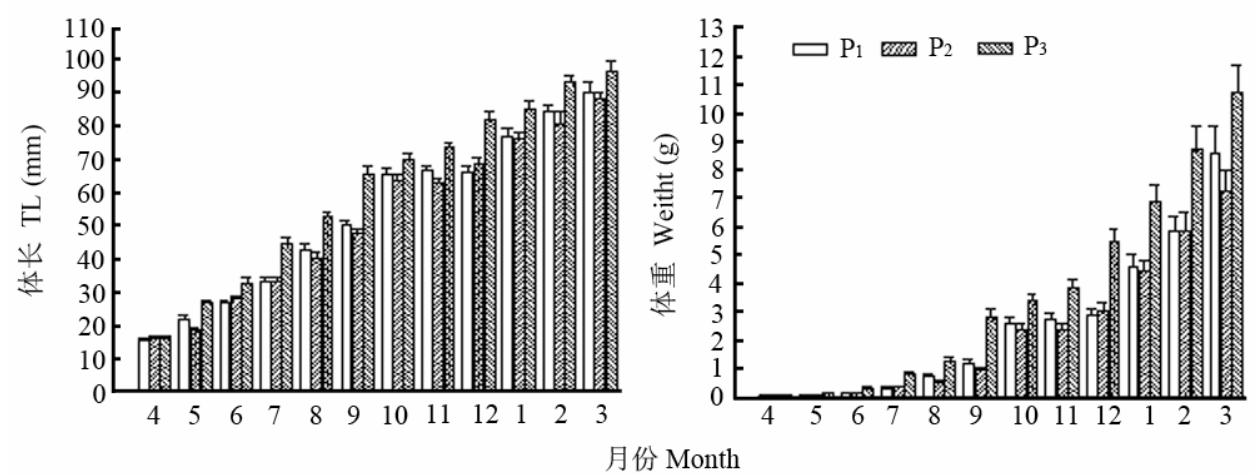

图 2 2008-2009 年滇池金线鲃稚鱼全长和体重的变化

Fig. 2 Total length and weight of Sinocyclocheilus grahami Juveniles from 2008 to 2009

开口时间也受到鱼卵的质量、卵黄囊的吸收和温度 的影响 (Yin, 1995)。进一步研究不同因素对滇池 金线鲃仔鱼开口时间的影响, 具有十分重要的实践 意义。

滇池金线鲃混合营养期 3 5d。随着卵黄囊的消 耗, 仔鱼身体的各个器官迅速发育完善, 很快就有 摄食活动, 所以其混合营养期比较短。这与初孵的 滇池金线鲃仔鱼的全长和卵黄囊较大有关。在此期 间, 仔鱼的受食能力迅速增强, 开口 3 5 d 后就能 够完全摄取外源性食物。及时提供充足饵料能够保 证仔鱼的品质，使其顺利进入稚鱼期。

\section{2 仔稚鱼的食性转化}

根据滇池金线鲃仔稚鱼食性转化的规律, 适时 采用浆状物一轮虫一人工混合饲料的方式, 可以提 高㱗料利用率, 减少水质污染, 从而大大提高滇池 金线鲃仔稚鱼的存活率。孵化一年后的滇池金线鲃 仔稚鱼存活率可达 $82.7 \%$ 。在自然环境下, 滇池金 线鲃仔稚鱼可以通过主动受食, 寻找到适合自己的 食谱, 而在养殖条件下, 由于养殖密度普遍较高,
天然食物的数量很难满足仔稚鱼的生长需要。活饵 虽然适口, 但是营养较单一, 如果不加强活饵的检 疫力度, 很容易增加鱼病的流行风险。此外, 在滇 池金线鲃仔稚鱼期间完全采用活诐饲养, 增加饲养 成本。仔稚鱼生长过程中, 需要经过几次食性转化, 所以很难用一种活饵就可以满足其摄食需要。而一 味地使用活饵饲养鱼苗存在转食成功率和成活率 偏低的问题。而配合饲料诱食性强, 营养全面; 成 系列的商业鱼饲料能够满足滇池金线鲃仔稚鱼不 同食性转换阶段对各种营养物质的需求。与鯁鿶白 鱼比较, 滇池金线鲃仔稚鱼具有很强的掠食性, 口 裂大, 可以很快地吞食较大颗粒的人工配合饲料。

\section{3 生长与密度的关系}

三种饲养密度, 滇池金线鲃仔鱼全长、体重和 SGR 的增长速度差别不大。这可能与仔鱼个体较 小, 摄食量少, 种内的竞争相对较小有关。同时, 在饲养管理中, 还通过不断加注新水改善水质, 减 少密度因子对仔鱼生长得制约。而滇池金线鲃稚鱼 以低密度饲养的全长、体重和 SGR 的增长速度最 
快。随着养殖密度的增高, 生长速度减慢。一般认 为养殖密度高, 种内在食物与空间的竞争加剧; 同 时, 仔稚鱼排出大量的代谢废物不能及时清除, 污 染水质, 使其生存环境的质量下降, 仔稚鱼的生长 受到抑制。低密度养殖的一些集群性鱼类, 由于被 分隔的个体产生古怪的不正常的行为, 食欲下降, 生长减慢; 当集群生活在一起时, 行动活泼, 摄食 积极, 生长加速 (Honer et al, 1987)。滇池金线鲃 稚鱼与史氏鲟 (Acipenser schrenckii) 鱼一样, 在试 验中没有出现低密度组中稚鱼生长减慢的现象 ( $\mathrm{Li}$ et al, 2004)。在养殖条件下, 一般很难引起种内激 烈的食物和空间上的竞争。密度制约往往通过水质 的改变影响稚鱼的生长。当提供溶氧充足和水温适 合的水质条件, 也许会减少负载对种群生长的影

\section{参考文献:}

Chen ZM, Yang JX, Su RF, Chen XY. 2001. Present status of the indigenous fishes in Dianchi lake, Yunnan [J]. Biodives Sci, 9(4): 407-413. [陈自 明, 杨君兴, 苏瑞风, 陈小勇. 2001. 滇池土著鱼类现状. 生物多样 性, 9(4): 407-413.]

Honer G, Roscnllml H, Khmer G. 1987. Growth of juvenile Sarotherodon galilaeus in laboratory aquaria [J] J Aquacul Tropics, 2(1): 59-71

Horváth L, Tamás G, Seagrave C. 2002. Carp and Pond Fish Culture. 2nd ed .[M]. Blackwell Scientific Publications Ltd., 1-170.

Li DP, Zhuang P, Yan AS, Wang MX, Zhang LZ. 2004. The influences of illumination, water current and stocking density on feeding, behaviour and growth in juveniles Acipenser schrenckii [J]. J Fish Chn, 28(1): 54-61. [李大鹏, 庄 平, 严安生, 王明学, 章龙珍. 2004. 光照、水 流和养殖密度对史氏鲟稚鱼摄食、行为和生长的影响. 水产学报, 28(1): 54-61.]

Li ZF, Chen SP, Zhuang YM, Zhuang JG. 2006. Studies on artificial propagation and seed breeding technology of Epinephelus lanceol [J]. Mar Fish Res, 27(3): 78-85. [黎祖福, 陈省平, 庄余谋, 庄杰贵. 2007. 鞍带石斑鱼人工繁殖与鱼苗培育技术研究. 海洋水产研究, 27(3): 78-85.]

Pan XF, Chen XY, Yang JX. 2009. Threatened fishes of the world: Sinocyclocheilus grahami (Regan) 1904 (Cyprinidae) [J]. Environ Biol fish, 85(1): 77-78.

Qiao ZG, Chang GL, Zhang JP, Niu JY, Wang W. 2007. Effect of delayed feeding on feeding ability, survival and growth of Silurus asotus larvae [J]. J Shanghai Fish Univ, 16(2): 130-134. [乔志刚, 常国亮, 张建平, 牛景彦, 王 武. 2007. 延迟投饵对鲇仔鱼摄食、存活和生长的影响.
响, 使其保持较稳定的生长率 (Schreck et al, 1985)。

\section{4 仔稚鱼饲养存在的问题}

从目前仔稚鱼的存活率和生长速度看, 滇池金 线鲃是一种优良的水产养殖品种。了解滇池金线鲃 食性转化和生长的特点, 可以有效地提高饲养效 率, 降低养殖成本。在培育鱼种时, 仔鱼阶段常常 发生气泡病和维生素 D 缺乏症, 造成大量死亡和导 致鱼苗品质下降。因此, 要注意管理水质和添加适 量维生素 D。在稚鱼阶段, 在季节转换时期, 常常 容易爆发小瓜虫和车轮虫病, 造成鱼苗大量死亡。 在 2008 年 10-11 月，3 个培育池的鱼苗生长速度 缓慢（图 2), 主要原因是 3 池爆发小瓜虫病所致; 与养殖水体的水温下降, 鱼苗摄食活动降低亦有一 定的关系。
上海水产大学学报, 16(2): 130-134.]

Schreck CB, Patino R, Pring CK, Winton JR, Holway JE. 1985. Effects of rearing density on indices of smoltification and performance of coho salmon, Oncorhynchus kisutch [J]. Aquaculture, 45(1-4): 345-358.

Yang JX, Pan XF, Li ZY. 2007. Preliminary report on the successful breeding of the endangered fish Sinocyclocheilus grahami endemic to Dianchi lake [J]. Zool Res, 28(3): 329-331. [杨君兴, 潘晓赋, 李再云. 2007. 云南滇池濒危特有种滇池金线鲃人工繁殖初报. 动物学研究, 28(3): 329-331.]

Yin MC. 1995. Ecology of Fishes [M]. Beijing: Agriculture of China Press, 1-293. [殷名称. 1995. 鱼类生态学. 北京: 中国农业出版社, 1-293.]

Youson JH. 1988. First metamorphosis [M]. Hoar WS, Randall DJ. 1988. Fish Physiology: XI. New York: Academic Press Inc, 135-196.

Yue PQ, Chen YY. 1998. China Red Data Book of Endangered Animals: Pisces[M]. Beijing: Science Press, 104-106. [乐佩琦, 陈宜瑜. 1998. 中国濒危动物红皮书(鱼类卷). 北京: 科学出版社, 104-106.]

Zhang BJ, Mo JH, Li BW, Li CZ, Huang LB, Zhang RY, Lu CS, Huang YQ, Liang RJ. 2007. Growth of larvae and juveniles of Oxyeleotris lineolatus and its seedlings culture in pond [J]. $J$ Guangdong Ocean Univ, 27(1): 27-33. [张邦杰, 莫介化, 李本旺, 李春枝, 黄林波, 张 瑞瑜, 陆昌胜, 黄永强, 梁仁杰. 2007. 线纹尖塘鳢仔幼鱼的生长发 育与池塘育苗研究. 广东海洋大学学报, 27(1): 27-33.]

Zhuang ZM, Wan RJ, Chen SP, Liu XF. 2005. Feeding and growth of the tonguefish Cynoglossus semilaevis larvae [J]. Acta Zool Sin, 51(6): 1023-1033. [庄志猛, 万瑞景, 陈省平, 刘新富. 2005. 半滑舌鲳的摄 食与生长. 动物学报, 51(6): 1023-1033.] 